\title{
Comparação entre diferentes softwares em um projeto de controle Fuzzy aplicado a uma cadeira de rodas motorizada
}

\author{
Luis F. F. Souza* Helena C. Maia* \\ Erivelton G. Nepomuceno* Márcio F. S. Barroso* \\ * Laboratório de Estudos em Controle e Modelagem, Departamento de \\ Engenharia Elétrica, Universidade Federal de São João del-Rei, MG, \\ (e-mails:lnandoffs@gmail.com, helena.6@outlook.com, \\ nepomuceno@ufsj.edu.br, barroso@ufsj.edu.br).
}

\begin{abstract}
Assistive Technologies exist to help people who can not perform their ordinary activities to reintegrate into the everyday world through the development of devices that meet the needs of people with functional diversity. Considering this, this work began with the application of questionnaires and interviews to wheelchair users in order to define the requirements of the control system for a motorized wheelchair. In this case, a Fuzzy control proposal was presented, which will be applied in an Arduino that will control a motorized wheelchair. For purposes of study and comparisons, it was proposed to implement this control in the Fuzzy Toolboxes of Matlab and LabVIEW. The proposed control was also implemented in Arduino and its response compared to the two Toolboxes. As a result, it was estimated the possible error generated in the implementation of the proposed control in Arduino according to LabVIEW and Matlab. The main conclusion of this work was that the two softwares analyzed were not sensitive to small changes in the defuzzification function, but Matlab presented more reliable results when compared to LabVIEW.

Resumo: As Tecnologias Assistivas existem para auxiliar pessoas que não conseguem realizar suas atividades corriqueiras a se reintegrarem ao mundo cotidiano, por meio do desenvolvimento de dispositivos que atendam certas necessidades de pessoas com diversidade funcional. Diante disso, esse trabalho se iniciou com a aplicação de questionários e entrevistas a cadeirantes com o intuito de definir os requisitos do sistema de controle para uma cadeira de rodas motorizada. Com isso, foi desenvolvida uma proposta de controle Fuzzy que será implementada em Arduino no qual controlará a cadeira de rodas motorizada. Para fins de estudo e comparações, foi proposta a implementação desse controle nos Toolboxes Fuzzy do Matlab e LabVIEW. A proposta de controle também foi implementada no Arduino e sua resposta comparada com os dois Toolboxes. Como resultado, foi estimado o erro possível gerado na implementação do controle proposto no Arduino de acordo com o LabVIEW e com o Matlab. A principal conclusão desse trabalho foi que os dois softwares analisados não são sensíveis a pequenas mudanças na função de defuzzyficação, porém o Matlab apresentou resultados mais confiáveis quando comparados ao LabVIEW.
\end{abstract}

Keywords: Fuzzy Control; Matlab; LabVIEW; Smart Control; Assistive Technology. Palavras-chaves: Controle Fuzzy; Matlab; LabVIEW; Controle Inteligente; Tecnologia Assistiva.

\section{INTRODUÇÃO}

Os trabalhos de Desai et al. (2017), Simpson (2005), Trefler et al. (2004) e Tefft et al. (1999) mostram que tanto crianças quanto adultos, que possuem alguma restrição de movimento, se beneficiam substancialmente do acesso a um meio de mobilidade independente, incluindo cadeiras de rodas motorizadas e manuais, scooters e andadores. Trabalhos envolvendo cadeiras de rodas motorizadas possuem o intuito de expandir o leque de deficiências atendidas pelo equipamento, pois nem todas possibilitam condições físicas ao cadeirante de fazer uso de uma cadeira de rodas

* Agradecemos à Fundação de Amparo a Pesquisa do Estado de Minas Gerais (FAPEMIG) e ao CNPq pelo apoio financeiro. manual e buscam outras alternativas, Leaman and La (2016). Devido a isso, é grande o número de pesquisas nessa vertente, como pode ser visto em Narin et al. (2018) e Leaman and La (2017).

Uma peça fundamental que faz parte deste equipamento é o controle, que consiste no caminho que leva a intenção de movimento do cadeirante até os atuadores do equipamento. De modo a trazer segurança e conforto ao cadeirante, é necessário que o controle seja preciso. Entre as diversas técnicas existentes, há o controle Fuzzy.

O controle Fuzzy se baseia na Lógica Fuzzy, esta proposta em 1965 por Lotfi Zadeh em Zadeh (1965) e que, diferente da lógica booleana, se baseia em graus de pertinência que 
podem assumir valores entre zero e um, ou seja, uma informação pode possuir um grau de pertinência entre falso e verdadeiro. O controle Fuzzy foi introduzido e aplicado pela primeira vez na década de 1970 em uma tentativa de projetar controladores para sistemas que são estruturalmente difíceis de modelar, Li and Gatland (1996). Desde então, tornou-se uma das áreas de pesquisa mais ativa na teoria dos conjuntos Fuzzy, resultando em muitas aplicações práticas para processos industriais, bem como estudos da própria teoria, Cai and Zhang (2008).

A lógica Fuzzy é usada no design de possíveis soluções para realizar navegação local, navegação global, planejamento de caminhos, controle de direção e de taxa de um robô móvel, Omrane et al. (2016). Essa lógica de controle provê um método de traduzir expressões verbais, vagas, imprecisas e qualitativas, comuns na comunicação humana em valores numéricos. Neste caso, traduzindo de forma compreensível para o computador o que é comum para o ser humano, Marçal and Susin (2005). Portanto, esse método se resume na busca em alcançar resultados que satisfaça à determinada situação especifica, como, por exemplo, no controle de posição do usuário de cadeira de rodas para definir a direção a ser seguida.

Uma das características mais fundamentais é que o controle Fuzzy é muito flexível em termos de adaptabilidade. Assim, com o uso do sistema baseado em Lógica Fuzzy, sensores caros e precisos não são necessários e sensores com baixa precisão e custo podem ser implementados, o que manterá o custo e a complexidade do sistema como um todo. Assim, o uso do controle Fuzzy torna a implementação do sistema muito mais prática, que é o objetivo número um, Tyagi et al. (2013). Devido a isso, o controle Fuzzy vem sendo utilizado em trabalhos de Tecnologias Assistivas, como pode ser visto em Jesus and Muñoz (2017), Guo et al. (2009) e Ghafari et al. (2006) e também em trabalhos envolvendo cadeiras de rodas motorizadas como pode ser visto em Rojas et al. (2018), Tyagi et al. (2013), Zal et al. (2013), Hong et al. (2012) e Islam et al. (2011).

Uma das formas de projetar o controle Fuzzy é por meio do software Matlab ou LabVIEW, entretanto ambos necessitam de um computador para sua utilização, o que inviabiliza seu uso para a implementação do controle Fuzzy em uma cadeira de rodas motorizada. A plataforma Arduino é uma opção embarcada bastante interessante por seu baixo custo, satisfatório processamento e sua baixa complexidade. Contudo, antes da implementação física é necessária a realização de simulações computacionais do controle Fuzzy para testes e possíveis ajustes.

Na literatura há trabalhos em que o controle Fuzzy é implementado no Toolbox do Matlab, tais como Aras et al. (2011), no qual o controle Fuzzy foi utilizado para controlar a temperatura durante o cozimento de arroz. O trabalho de Martínez et al. (2010) também utiliza esse Toolbox, neste o objetivo é identificar as qualidades de indivíduos para formar equipes de trabalho mais adequadas. No trabalho de Tyagi et al. (2013), a implementação do controle Fuzzy proposto para uma cadeira de rodas também foi simulado neste Toolbox. Entretanto, não há indícios na literatura de uma investigação entre os resultados obtidos pelo Matlab e outros softwares. Neste trabalho é proposto a Lógica Fuzzy para realizar o controle de uma cadeira de rodas motorizada, considerando a entrada proveniente de um joystick. Diante dessa implementação, foi proposta uma comparação entre os resultados obtidos pelo Toolbox Fuzzy do Matlab e do LabVIEW, além da comparação dos mesmos resultados com a resposta obtida pela biblioteca eFLL (Embedded Fuzzy Logic Library), que permite a implementação do controle Fuzzy no Arduino.

\section{OBJETIVOS}

Este trabalho propõe um estudo da resposta do controle Fuzzy utilizando os softwares Matlab e LabVIEW. O intuito é analisar qual dos dois se aproxima mais do resultado analítico proveniente da defuzzyficação, a última etapa do controle Fuzzy que gera a saída. Além disso, esse trabalho propõe um controle Fuzzy para a movimentação de uma cadeira de rodas de acordo com o que foi analisado em entrevistas realizadas com cadeirantes.

De forma a averiguar o resultado do controle proposto, foi estimado o erro possível gerado na resposta do Arduino ao implementar esse controle. Foram encontradas duas estimações, de acordo com os dois softwares, que foram comparadas entre si levando em conta o estudo previamente realizado, cuja finalidade foi a de mostrar qual dos dois se aproxima mais da reposta analítica.

O controle proposto nesse trabalho não foi implementado em uma cadeira de rodas em tamanho real, apenas na plataforma Arduino com entradas provenientes de vetores previamente declarados com valores variando de 100 a 900 que simulam os valores provenientes de um joystick.

\section{METODOLOGIA}

A seguir, são apresentadas as etapas do trabalho.

\subsection{Entrevistas}

A primeira etapa do trabalho foi a realização de um estudo que visou entender quais são os aspectos ergonômicos e de controle mais importantes para realizar um projeto de cadeira de rodas motorizada.

Com base no estudo realizado, no levantamento das informações e com o apoio de um cadeirante colaborador do projeto Paramec-UFSJ, foi elaborado um roteiro de entrevistas e estruturado um questionário contendo quinze questões abertas. Foram realizadas entrevistas com o objetivo de identificar as principais características e queixas dos cadeirantes em relação ao conforto, controle, segurança e bem estar, quanto aos modelos convencional e motorizado de cadeira de rodas, disponíveis no mercado.

As entrevistas foram aplicadas a sete cadeirantes da cidade de São João del-Rei, Minas Gerais. Posteriormente, foi realizada uma análise e, com isso, criou-se um questionário para aplicação em nível nacional. O questionário foi disponibilizado em fóruns e redes sociais com participação de cadeirantes, o qual foi respondido por 60 pessoas. A partir desses resultados coletados e das respectivas análises qualitativas e quantitativas, foram extraídos os principais desafios que os cadeirantes enfrentam atualmente.

Após esse estudo, concluiu-se que o sistema de controle da cadeira de rodas deveria apresentar características suaves, 
ou seja, sem a presença de movimentos bruscos, pois são prejudiciais a saúde e ao conforto dos cadeirantes. Dentre as técnicas de controle existentes na literatura, foi proposta a implementação da Lógica Fuzzy.

\subsection{Controle Fuzzy: Exemplo de comparação}

O controle Fuzzy possui três etapas:

- Fuzzyficação: Etapa que converte os valores de entrada em graus de pertinência entre 0 e 1.

- Inferência: Etapa que especifica a reação a cada ação para o controle desejado por meio de um conjunto de regras de pertinência SE-ENTÃO.

- Defuzzyficação: Etapa responsável por encontrar a saída numérica do controle, ou seja, ocorre a transformação dos graus de pertinência provenientes da fuzzyficação em valores de saída.

Por meio de um exemplo simples, serão apresentados os resultados obtidos pelo Toolbox Fuzzy do LabVIEW e do Matlab. Para tal comparação, foram implementadas as funções de pertinência mostradas na Figura 1, onde a fuzzyficação está ligada à função de pertinência Entrada e a defuzzyficação à função Saída.
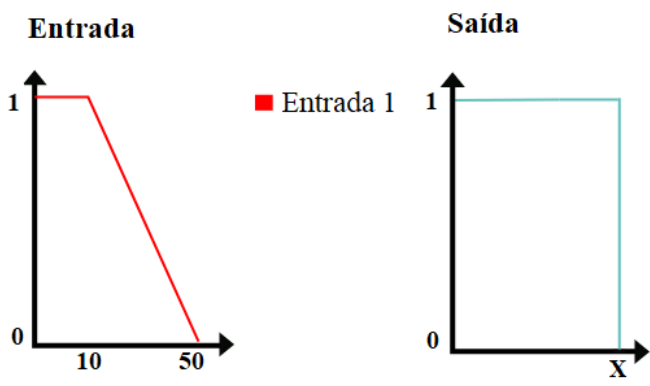

- Saída 1

Figura 1. Funções de pertinência: Entrada e Saída.

Cuja regra de pertinência aplicada é:

\section{Se Entrada é Entrada 1 então Saída é Saída 1}

Com o intuito de definir uma referência para a comparação entre os dois Toolboxes, foi proposta no Matlab uma rotina que retorna o resultado analítico do controle Fuzzy. Para isso, é necessário escolher o método de defuzzyficação a ser utilizado nos softwares. Neste trabalho foi empregada a técnica do Centroide.

Segundo Marro et al. (2010), a fórmula matemática para o cálculo do centroide é expressa na equação (1).

$$
C O G=\frac{\sum_{x=a}^{b} \mu(x) \cdot x}{\sum_{x=a}^{b} \mu(x)}
$$

Onde COG é o sinal de saída, $a$ e $b$ são os valores mínimo e máximo da função de pertinência de defuzzyficação, $x$ é o valor da função de pertinência no ponto $y$, ponto do valor do grau de pertinência, e $\mu(x)$ é o próprio valor de $y$.

A função "Saída1" de defuzzyficação possui o formato geométrico de um retângulo, como pode ser visto na Figura 1. A rotina foi utilizada para encontrar o centroide desse retângulo. Neste caso, o passo de iteração da estrutura for é igual a 0.1. Porém, para esse caso específico, o cálculo do centroide não depende do passo de iteração.

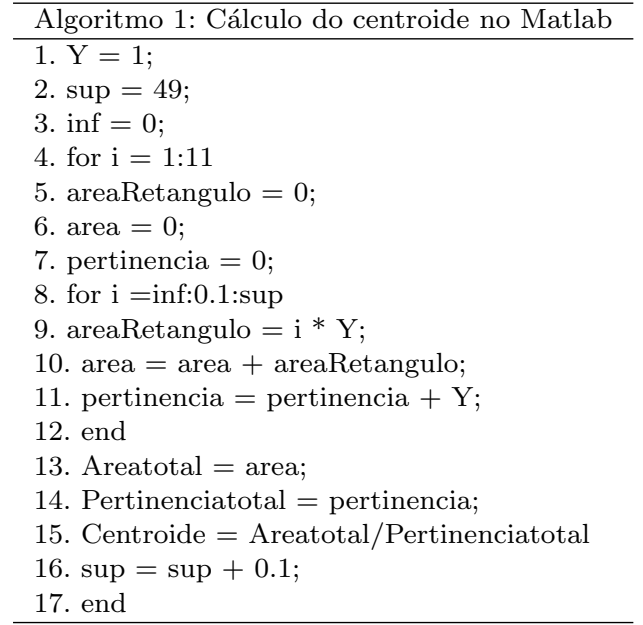

$\mathrm{Na}$ rotina acima a equação (1) foi implementada, onde o dividendo é calculado na variável area e o divisor em pertinencia. Além disso, o valor do grau de pertinência, atribuída na variável $Y$, é igual a 1 , considerando que a entrada varia de 0 a 10 , como pode ser visto na Figura 1. Ao analisar a entrada variando de 10 a 50 o valor de pertinência é variável. Entretanto, devido ao formato retangular da função "Saída 1", o valor do centroide será sempre o mesmo independente do valor de $Y$.

Com o intuito de comparar os resultados obtidos nos softwares LabVIEW e Matlab, esse trabalho propõe variar a largura do retângulo da função de defuzzyficação "Saída 1 ", que está contida no intervalo de 0 a até a variável $X$. Nesse caso, a variável $X$ foi substituída por valores entre 49 e 50, como ilustra a Tabela 1 . A segunda coluna dessa tabela apresenta as respostas obtidas pela rotina.

Tabela 1. Comparação entre os resultados dos softwares.

\begin{tabular}{cccc} 
Valor de x & Rotina & Matlab & LabVIEW \\
\hline 49 & 24,5 & 24,5 & 24,8253 \\
49,1 & 24,55 & 24,5 & 24,8253 \\
49,2 & 24,60 & 24,5 & 24,8253 \\
49,3 & 24,65 & 24,5 & 24,8253 \\
49,4 & 24,70 & 24,5 & 24,8253 \\
49,5 & 24,75 & 24,5 & 24,8253 \\
49,6 & 24,80 & 24,5 & 24,8253 \\
49,7 & 24,85 & 24,5 & 24,8253 \\
49,8 & 24,90 & 24,5 & 24,8253 \\
49,9 & 24,95 & 24,5 & 24,8253 \\
50 & 25 & 25 & 24,8253 \\
\hline
\end{tabular}

3.3 Controle Fuzzy para uma cadeira de rodas motorizada

A seguir serão apresentadas as etapas do controle Fuzzy proposto para uma cadeira de rodas motorizada.

\subsubsection{Fuzzyficação}

Nesta proposta, a entrada vem de um joystick. Ele possui dois eixos, X e Y. O eixo X coleta os movimentos dos lados: direita e esquerda, já o eixo Y os movimentos para frente.

A Figura 2 mostra as funções de pertinência para a fuzzyficação dos dois eixos. Caso o joystick esteja em repouso, então a pertinência será máxima, ou seja, igual a 1, para as funções PararX e PararY. Neste caso foi definido o ponto de repouso do joystick, sendo igual a 500 . 


\section{Eixo X}

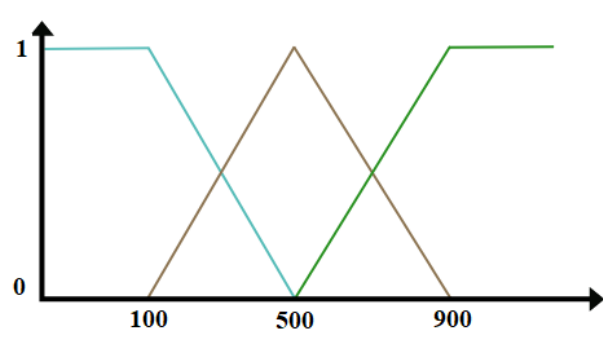

Eixo Y

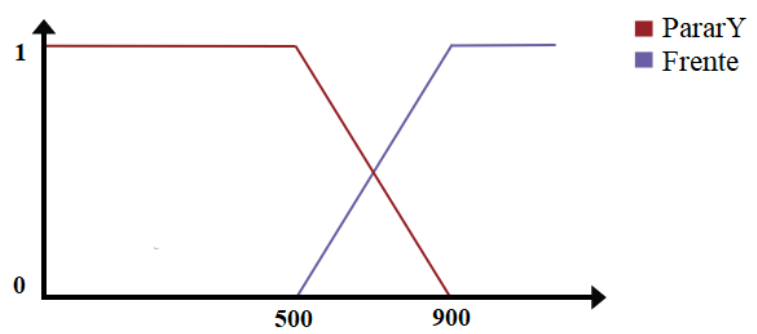

Figura 2. Funções para fuzzyficação.

Na Figura 2 o eixo x representa a entrada que, por ser um joystick analógico, varia de 0 a 1023. Já o eixo y representa os graus de pertinência aos quais a entrada será convertida.

Interpretando a Figura 2, ela indica que a medida que o valor de entrada seja menor que 500, o Joystick está sendo movimentado para o lado "Esquerda", já o contrário, indica o lado "Direita".

\subsubsection{Inferência}

A inferência foi composta por regras que determinam qual será a direção que a cadeira de rodas irá se deslocar ao ser controlada pelo joystick. Elas são apresentadas a seguir, respectivamente, para o motor esquerdo e direito.

Tabela 2. Regras de pertinência para o motor esquerdo.

\begin{tabular}{cccccc} 
Regra & Direção & Eixo x & $\begin{array}{c}\text { Associação } \\
\text { entre x e y }\end{array}$ & Eixo y & Saída \\
\hline 1 & Direita & Direita & & Parar & Rápido 1 \\
2 & $\begin{array}{c}\text { Esquerda } \\
\text { Esquerda }\end{array}$ & Parar & Inverte \\
3 & $\begin{array}{c}\text { Diagonal } \\
\text { Direita }\end{array}$ & Direita & AND & Frente & Rápido 2 \\
& Diagonal & Esquerda & & Frente & Devagar \\
4 & Esquerda & Parar & & Frente & Rápido 3 \\
5 & Frente & Parar \\
\hline
\end{tabular}

Tabela 3. Regras de pertinência para o motor direito.

\begin{tabular}{cccccc} 
Regra & Direção & Eixo x & $\begin{array}{c}\text { Associação } \\
\text { entre x e y }\end{array}$ & Eixo y & Saída \\
\hline 1 & Direita & Direita & & Parar & Inverte \\
2 & $\begin{array}{c}\text { Esquerda } \\
\text { Esquerda }\end{array}$ & Parar & Rápido 1 \\
3 & $\begin{array}{c}\text { Diagonal } \\
\text { Direita }\end{array}$ & Direita & AND & Frente & Devagar \\
& Diagonal & Esquerda & & Frente & Rápido 2 \\
4 & Esquerda & Parar & & Frente & Rápido 3 \\
5 & Frente & Parar \\
\hline 6 & Parar & Parar & & Parar & Parar \\
\hline
\end{tabular}

A proposta de controle foi implementada no Matlab e do LabVIEW. Em ambos os casos os dois vetores de entrada variaram de 100 a 900, de uma em uma unidade. Com isso, foi gerado o gráfico da Figura 4. Esse gráfico ilustra o comportamento da tensão de um motor de 24 V. Segundo McRoberts (2011) para se obter o equivalente em tensão do sinal enviado ao motor pela plataforma Arduino, é necessário dividir o valor por 255 e multiplicar pelo valor máximo de tensão, que, neste caso, é $24 \mathrm{~V}$. Os valores negativos de tensão indicam rotação no sentido anti-horário. O gráfico foi obtido utilizando o software Matlab.

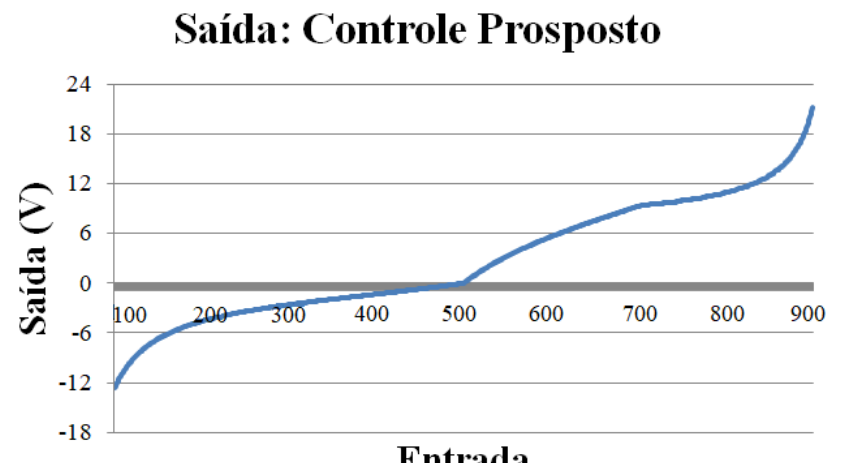

Entrada

Figura 4. Saída em tensão do motor esquerdo.

Neste gráfico visualiza-se as seguintes regras em ação: 
- Entrada 100 a 300: As funções de fuzzyficação Esquerda e PararY ativam a regra 2. Por consequência, o motor irá girar no sentido anti-horário.

- Entrada 300 a 700: As funções de fuzzyficação PararX e PararY estão acionadas ativando a regra 6. Logo, o motor poderá girar no sentido anti-horário ou horário.

- Entrada 700 a 900: As funções de fuzzyficação Direita e Frente estão acionadas ativando a regra 3. Por consequência, o motor irá girar no sentido horário.

Já para o motor direito, o comportamento é o da Figura 5.

\section{Saída: Controle Prosposto}

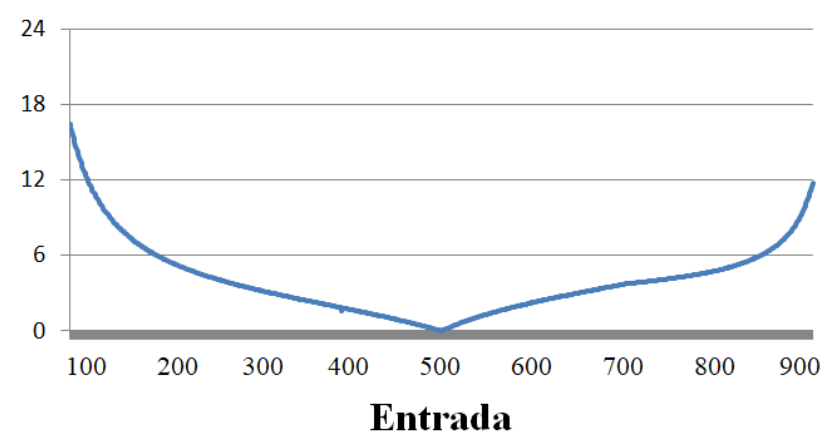

Figura 5. Saída em tensão do motor direito.

Posteriormente, os vetores de saída obtidos foram comparados entre si e com o resultado proveniente do Arduino.

\subsection{Comparação entre os resultados obtidos}

O resultado do módulo da diferença entre os dois vetores de saída gerados pelos softwares é mostrado na Figura 6.

\section{Diferença: Matlab e LabVIEW}

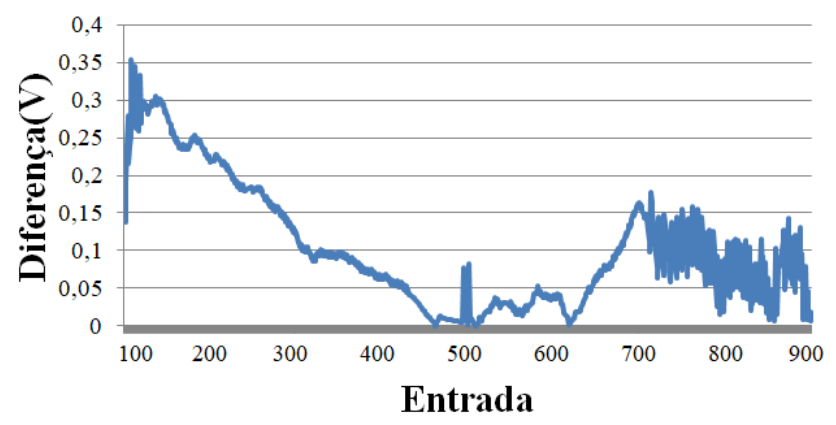

Figura 6. Diferença em volts (V) entre os vetores de saída do Matlab e do LabVIEW.

Neste caso, pode-se perceber que, para valores baixos de entrada, a diferença entre os softwares foi maior do que quando comparado para valores altos de entradas.

\subsection{Comparação com o resultado obtido no Arduino}

O controle proposto neste trabalho também foi implementado no Arduino, utilizando a biblioteca eFLL, de forma a ilustrar a diferença de resultados entre os dois softwares.

A Figura 7 mostra o resultado obtido pela subtração do vetor de saída do Matlab pelo vetor de saída do Arduino.

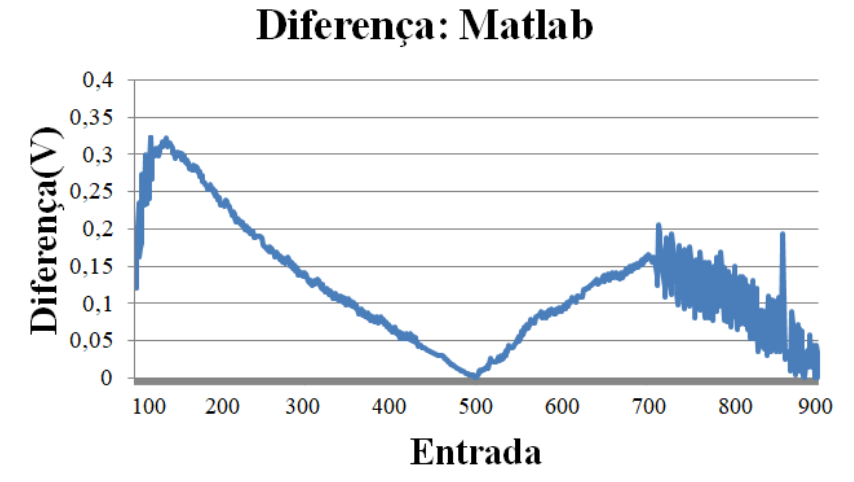

Figura 7. Diferença em volts (V) entre os vetores de saída do Matlab e do Arduino.

Já a Figura 8 ilustra o resultado obtido pela subtração do vetor de saída do LabVIEW pelo vetor de saída do Arduino.

\section{Diferença: LabVIEW}

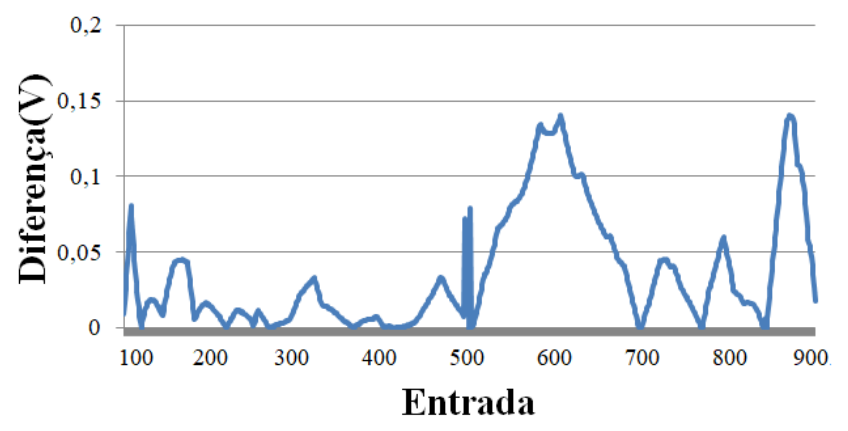

Figura 8. Diferença em volts (V) entre os vetores de saída do LabVIEW e do Arduino.

\section{DISCUSSÃO DOS RESULTADOS}

Dentre todas as técnicas de controle, o controle Fuzzy foi escolhido por sua facilidade de ajuste e por não necessitar de um modelo matemático do sistema. Alem disso, de acordo com o relatado pelos cadeirantes, essa técnica se mostra promissora.

Com os resultados da Tabela 1 conclui-se que os dois softwares não apresentam sensibilidade para pequenas mudanças na função de defuzzyficação. No exemplo da Tabela 1, o Matlab truncou os valores de 0,5 a 0,5 e o LabVIEW apresentou uma mesma resposta em todos os valores. Logo, é possível afirmar que o Matlab apresentou resultados mais confiáveis, pois o seu resultado se aproxima mais do analítico obtido pela rotina que calcula o centroide. Portanto, a referência a ser utilizada em trabalhos futuros será o software Matlab.

Já o resultado obtido com a proposta de controle da cadeira de rodas apresentou diferença entre os dois softwares superior a $0,3 \mathrm{~V}$, que é um valor considerável, mas não relevante para um motor de $24 \mathrm{~V}$. A comparação do controle proposto no Arduino com os Toolboxes também mostrou uma diferença relevante entre os dois softwares. Ao analisar o Matlab e o Arduino, obteve-se um erro máximo de 1,4\%. Por outro lado, realizando a comparação 
entre o LabVIEW e Arduino, esse erro máximo foi de 0,55\%. Essa porcentagem considera o valor máximo de saída do Arduino, que varia de 0 a 255.

\section{CONCLUSÕES FINAIS}

Em trabalhos futuros, o controle Fuzzy proposto neste trabalho controlará uma cadeira de rodas por meio de um Arduino utilizando outro método de implementação. É necessário outro método porque, segundo Kridi et al. (2011), a biblioteca eFLL suporta apenas uma saída e para esse projeto são necessárias duas, pelo fato da cadeira de rodas possuir dois motores, ou seja, duas saídas. Logo, será usado o software Matlab para visualizar se a diferença entre as respostas obtidas pelo Arduino e Matlab está aceitável, pois se o erro for alto, a cadeira pode não seguir corretamente a intenção de movimento do cadeirante. Também serão estudadas possíveis influências para o desempenho do controle, como, por exemplo, a porcentagem de bateria disponível. Além disso, com o protótipo em mãos o controle Fuzzy será comparado a outras técnicas de controle, de forma a avaliar o desempenho das técnicas disponíveis na literatura.

\section{AGRADECIMENTOS}

À Fundação de Amparo a Pesquisa do Estado de Minas Gerais (FAPEMIG), à CAPES, ao CNPq pelo apoio financeiro e à Universidade Federal de São João del-Rei (UFSJ) pelo apoio logístico e infraestrutura física.

\section{REFERÊNCIAS}

Aras, M., Ali, F., Azis, F., Hamid, S., and Basar, M. (2011). Performances evaluation and comparison of two algorithms for fuzzy logic rice cooking system (matlab fuzzy logic toolbox and fuzzytech). IEEE Conference on Open Systems, 400-405.

Cai, K. and Zhang, L. (2008). Fuzzy reasoning as a control problem. IEEE Transactions on Fuzzy Systems, 600614.

Desai, S., Mantha, S.S., and Phalle, V.M. (2017). Advances in smart wheelchair technology. 2017 International Conference on Nascent Technologies in the Engineering Field (ICNTE-2017), 1-7.

Ghafari, A., Meghdari, A., and Vossoughi, G. (2006). Intelligent control of powered exoskeleton to assist paraplegic patients mobility using hybrid neuro-fuzzy anfis approach. IEEE International Conference on Robotics and Biomimetics, 733-738.

Guo, H., Cao, C., Yang, J., and Zhang, Q. (2009). Research on obstacle-avoidance control algorithm of lower limbs rehabilitation robot based on fuzzy control. Sixth International Conference on Fuzzy Systems and Knowledge Discovery, 151-155.

Hong, T., Nakhaeinia, D., and Karasfi, B. (2012). Application of fuzzy logic in mobile robot navigation. In E. Dadios (ed.), Fuzzy Logic - Controls, Concepts, Theories and Applications, chapter 2, 21-36. InTech.

Islam, S., Sarker, M., Ali, S., Othman, M., and Majlis, B. (2011). Design, simulation and synthesis of wheelchair controller using fuzzy logic. IEEE Regional Symposium on Micro and Nano Electronics, 117-122.
Jesus, T. and Muñoz, D. (2017). Controle fuzzy embarcado em arquiteturas reconfiguráveis para desvio de obstáculos em sistema de navegação para deficientes visuais. In Simpósio Brasileiro de Automação Inteligente, volume XIII. Porto Alegre.

Kridi, D., Alves, A., Lemos, M., and Rabêlo, R. (2011). Desenvolvimento de uma biblioteca fuzzy para o controle autônomo de um robô móvel em ambiente desconhecido. Mostra Nacional de Robótica, I.

Leaman, J. and La, H.M. (2016). Development of a smart wheelchair for people with disabilities. 2016 IEEE International Conference on Multisensor Fusion and Integration for Intelligent Systems (MFI 2016), 279284.

Leaman, J. and La, H. (2017). A comprehensive review of smart wheelchairs: Past, present, and future. IEEE Transactions on Human-Machine systems, 486-499.

Li, H. and Gatland, H. (1996). Conventional fuzzy control and its enhancement. IEEE Transactions on Systems, Man, and Cybernetics - Part B: Cybernetics, 791-797.

Marro, A., Souza, A., Cavalcante, E., Bezerra, G., and Nunes, R. (2010). Lógica fuzzy: Conceitos e aplicações. Universidade Federal do Rio Grande do Norte, 23p.

Martínez, L., Licea, G., Rodríguez, A., Castro, J., and Castillo, O. (2010). Using matlab's fuzzy logic toolbox to create an application for ramset in software engineering courses. Computer Applications in Engineering Education, 596-605.

Marçal, R. and Susin, A. (2005). Detectando falhas incipientes em máquinas rotativas. Revista Gestão Industrial, 01, 083-092.

McRoberts, M. (2011). Arduino Básico. Novatec, São Paulo.

Narin, B., Brian, M., and Smart, W.D. (2018). A critical look at smart wheelchairs. arXiv:1809.00291.

Omrane, H., Masmoudi, M.S., and Masmoudi, M. (2016). Fuzzy logic based control for autonomous mobile robot navigation. Computational Intelligence and Neuroscience, 2016, 1-10.

Rojas, M., Ponce, P., and Molina, A. (2018). A fuzzy logic navigation controller implemented in hardware for an electric wheelchair. International Journal of Advanced Robotic Systems, 15.

Simpson, R. (2005). Smart wheelchairs: A literature review. Journal of Rehabilitation Research \& Development, 423-436.

Tefft, D., Guerette, P., and Furumasu, J. (1999). Cognitive predictors of young children's readiness for powered mobility. Dev Med Child Neurol, 41, 665-670.

Trefler, E., Fitzgerald, S., Hobson, D., Bursick, T., and Joseph, R. (2004). Outcomes of wheelchair systems intervention with residents of long-term care facilities. Assist Technol, 1, 18-27.

Tyagi, V., Gupta, N., and Tyagi, P. (2013). Smart wheelchair using fuzzy inference system. IEEE GHTC$S A S, 175-180$.

Zadeh, L.A. (1965). Fuzzy sets. Information and Control, 08, 338-353.

Zal, F., Chen, T., Chi, S., and Kuo, C. (2013). Fuzzy controller based subsumption behavior architecture for autonomous robotic wheelchair. International Conference on Advanced Robotics and Intelligent Systems, 158-163. 